\title{
A crossmodal attentional blink between vision and touch
}

\author{
SALVADOR SOTO-FARACO \\ University of British Columbia, Vancouver, British Columbia, Canada \\ CHARLES SPENCE and KATHERINE FAIRBANK \\ University of Oxford, Oxford, England \\ ALAN KINGSTONE \\ University of British Columbia, Vancouver, British Columbia, Canada \\ ANNE P. HILLSTROM \\ University of Texas, Arlington, Texas \\ and \\ KIMRON SHAPIRO \\ University of Wales, Bangor, Wales
}

\begin{abstract}
There is currently a great deal of interest regarding the possible existence of a crossmodal attentional blink (AB) between audition and vision. The majority of evidence now suggests that no such crossmodal deficit exists unless a task switch is introduced. We report two experiments designed to investigate the existence of a crossmodal $\mathrm{AB}$ between vision and touch. Two masked targets were presented successively at variable interstimulus intervals. Participants had to respond either to both targets (experimental condition) or to just the second target (control condition). In Experiment 1, the order of target modality was blocked, and an $\mathrm{AB}$ was demonstrated when visual targets preceded tactile targets, but not when tactile targets preceded visual targets. In Experiment 2, target modality was mixed randomly, and a significant crossmodal $\mathrm{AB}$ was demonstrated in both directions between vision and touch. The contrast between our visuotactile results and those of previous audiovisual studies is discussed, as are the implications for current theories of the $\mathrm{AB}$.
\end{abstract}

When people search for two predefined visual targets within a rapid sequence of visual stimuli, selection of the first target (T1) is often followed by a temporary reduction in the ability to identify or detect the second target (T2). This phenomenon, known as the attentional blink (AB; Raymond, Shapiro, \& Arnell, 1992), has been characterized as a temporary interference in stimulus encoding that renders T2 unavailable for recall (e.g., Duncan, Martens, \& Ward, 1997; Duncan, Ward, \& Shapiro, 1994; Potter, Chun, Banks, \& Muckenhoupt, 1998; Raymond et al., 1992; Shapiro, Raymond, \& Arnell, 1994; R. Ward, Duncan, \& Shapiro, 1996, 1997). Although the majority of $\mathrm{AB}$ studies are purely visual, several authors have shown that $\mathrm{AB}$ can occur in the auditory (Arnell \& Jolicœur,

This research was partially supported by a Network Grant from the McDonnell-Pew Centre in Oxford. We thank Vince Di Lollo for insightful comments on an earlier version of the manuscript, Thomas Hope for help in the testing phase, and Xavier Mayoral for programming assistance with Expe6. Correspondence concerning this article should be addressed to S. Soto-Faraco, Departament de Psicologia Bàsica, Universitat de Barcelona, P. Vall d'Hebrón, 171, 08035-Barcelona, Spain (e-mail: ssoto@psico.psi.ub.es).
1999; Duncan et al., 1997; Soto-Faraco \& Spence, 2002) and tactile (Hillstrom, Shapiro, \& Spence, 2002) modalities. In Hillstrom et al.'s study, two masked tactile targets were presented in rapid succession to the index or ring finger among a stream of distractors presented to the middle finger. Overall, localization performance on T2 was inversely related to the T1-T2 interstimulus interval (ISI), but this temporary interference was larger when T1 had to be responded to than when it could be ignored, suggesting the existence of a tactile AB.

One of the most controversial aspects of the $A B$ is whether it occurs between targets in different sensory modalities (e.g., Arnell \& Duncan, 2002; Arnell \& Jolicœur, 1999; Duncan et al., 1997; Potter et al., 1998; SotoFaraco \& Spence, 2002). To date, all crossmodal AB research has focused solely on selection between auditory and visual targets. In the present study, we investigated whether a crossmodal $\mathrm{AB}$ exists between vision and touch.

Several studies have shown AB-like interference between auditory and visual targets (e.g., Arnell \& Jolicœur, 1999; Jolicœur, 1999; Jolicœur \& Dell' Acqua, 1999), whereas others have found no evidence of an audiovisual 
AB (e.g., Duncan et al., 1997; Potter et al., 1998; SotoFaraco \& Spence, 2002). Many researchers now believe that task-switching 1 may explain these differences (e.g., Potter et al., 1998; Soto-Faraco \& Spence, 2002). Specifically, an audiovisual AB-like interference effect is often observed when different tasks are associated with each target modality (i.e., identification of a digit, T1, in a visual stream, and subsequent detection of an " $X$," T2, in a concurrent stream of spoken letters; Arnell \& Duncan, 2002; Arnell \& Jolicœur, 1999; Jolicœur, 1999), suggesting that task-switching may be necessary (although probably not sufficient; see Duncan et al., 1997) to ensure the occurrence of an AB. Conversely, when modalityswitching is not confounded with task-switching, no audiovisual AB occurs (Potter et al., 1998; Soto-Faraco $\&$ Spence, 2002).

It appears that for the audiovisual case, the selection of a target in one modality does not necessarily interfere with the selection of a subsequent target in a different modality, unless participants also have to switch from one task to another. However, it is unclear whether the same conclusion would hold for attentional selection between other pairs of sensory modalities. Indeed, extensive crossmodal links in spatial attention have been demonstrated between touch and vision (e.g., Spence, Nicholls, Gillespie, \& Driver, 1998; Spence, Pavani, \& Driver, 2000). Given the recent debate concerning the nature of crossmodal links in exogenous (stimulus-driven) attention between different pairs of sensory modalities (e.g., Spence \& Driver, 1997a; L M. Ward, 1994; see Spence, 2001, for a review) and the unique role that each sensory modality plays in providing information about the environment (Hughes, 1999; Stein \& Meredith, 1993), the most appropriate strategy is to examine each crossmodal interaction separately.

In the present study, we used a crossmodal visuotactile version of the two-target paradigm, in which two masked targets are presented at variable ISIs. This method provides a reliable measure of AB (see, e.g., Duncan et al., 1994; R. Ward et al., 1997) in the analysis of the time course of performance for the second target as a function of whether or not the first target has to be attended (i.e., whether or not it requires a response). We used a spatial localization task for each target because numerous studies have shown that attentional effects are more pronounced when the tasks involve some form of spatial discrimination (e.g., Hillstrom et al., 2002; McDonald \& Ward, 1999; Spence \& Driver, 1994; Spence et al., 2000). However, it is worth noting that the task in the present experiments (target localization) was the same for both modalities. Therefore, in contrast with previous studies in which a crossmodal $\mathrm{AB}$ was reported, potential interference effects between $\mathrm{T} 1$ and $\mathrm{T} 2$ cannot reflect an artifact of task-switching.

Our working hypothesis was that, if the attentional selection of a stimulus (T1) in one modality produces a temporary interference in the ability to select a subsequent target (T2) in another modality, then a time-locked decrement should be observed on $\mathrm{T} 2$ performance when par- ticipants have to respond to both targets. The control condition ("ignore T1 and respond to T2 only") was intended to provide a baseline measure of T2 accuracy when $\mathrm{T} 1$ need not be attended.

\section{EXPERIMENT 1}

\section{Method}

Participants. Fourteen undergraduate students at the University of Oxford participated in this experiment. They were divided into two groups according to the order in which the targets were presented (visual-tactile, $n=8$; tactile-visual, $n=6$ ). All had normal or corrected-to-normal vision and reported normal touch, and all performed at over $50 \%$ correct in the control condition. The Experiment lasted approximately $75 \mathrm{~min}$.

Apparatus and Materials. Nine yellow LEDs arranged in a $3 \times$ 3 array were attached to one face of a cube $(9 \mathrm{~cm} \times 9 \mathrm{~cm} \times 9 \mathrm{~cm})$. One tactile stimulator (Oticon-A bone-conduction vibrator with a vibrating surface of $1.6 \mathrm{~cm} \times 2.4 \mathrm{~cm}$ ) was attached to each of the four front corners of the cube, on the side of the corresponding LED (topleft, top-right, bottom-left, and bottom right). When activated, the tactile stimulators delivered a $200-\mathrm{Hz}$ vibration at comfortable suprathreshold intensity. Four foot pedals placed on the floor were used to collect responses. The LEDs and vibrators, as well as the registration of foot-pedal responses, were controlled by a PC-driven relay box through a DOP-24 and a DCM-16 board (Blue Chip Technology, Deeside, Scotland). The experimental protocol was programmed using the Expe6 software (Pallier, Dupoux, \& Jeannin, 1997).

Figure 1 illustrates the sequence of events on a trial. Each trial started with all LEDs except the central one turned on and the vibrators turned off. Visual targets consisted of the blink of one of the four corner LEDs (32 msec off: $18 \mathrm{msec}$ on), followed by a visual mask that was composed of four consecutive 25-msec arrangements of the LED display. The first and third configurations consisted of switching off the four LEDs at the corners plus the central LED, and switching on the rest. The second and fourth configurations consisted of switching on a random subset of LEDs and switching off the rest. ${ }^{2}$ Tactile targets consisted of the onset of one of the vibrators for $40 \mathrm{msec}$, a 10 -msec still interval, and then all four vibrators activated for $100 \mathrm{msec}$ (as the tactile mask).

Procedure. The participants were seated in a dark, soundproof booth, with their feet depressing the four foot pedals (located beneath the toes and heels of both feet). They held the cube with the LED display facing toward them (index finger and thumb of each hand on the top and bottom vibrators, respectively, of each side of the cube). A loudspeaker cone delivered constant white noise in front of the participants at an intensity sufficient to mask the sound produced by the vibrators.

On each trial, the participants were presented with a succession of two masked targets (visual-tactile for the VT group and tactilevisual for the TV group) separated by a variable $\operatorname{ISI}^{3}(300,450,750$ or 1,500 $\mathrm{msec}$ ). Each target position (four possible) and ISI was equiprobable and randomly selected from trial to trial. The participants responded by releasing one foot pedal in a manner congruent with the location of the target (i.e., right toe for top right corner, right heel for bottom right corner, left toe for top left corner, and left heel for bottom left corner). An additional red LED, placed on the top of the cube, was illuminated whenever a foot pedal response was registered. After the response(s), a 1,500-msec interval led to the next trial. All participants were presented with four blocks of 128 trials (two experimental and two control), run in alternation with order counterbalanced. In the experimental blocks, the participants responded to both targets in the order of presentation. In the control blocks, the participants responded only to the second target (which was tactile or visual, depending on the group).

Three short practice blocks were presented prior to the experiment. The first contained 8 single nonmasked target trials ( 4 in each 

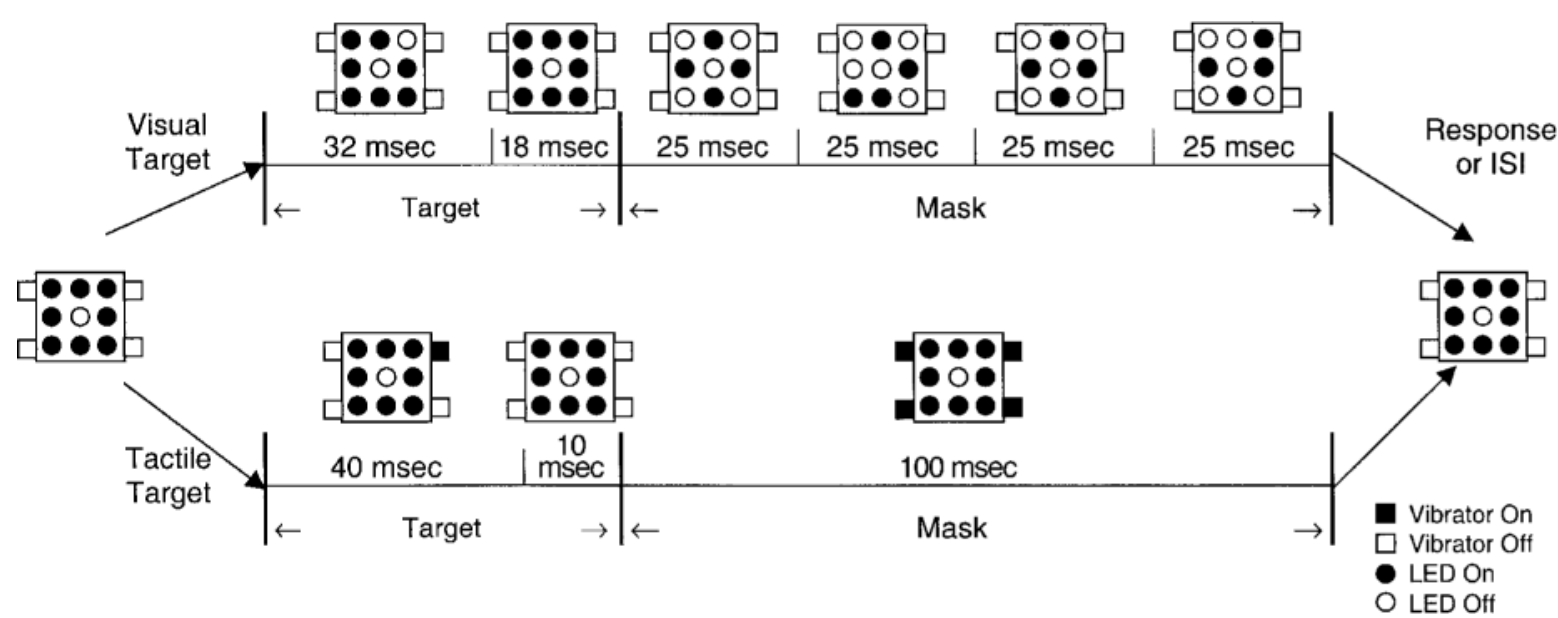

Figure 1. Temporal sequence of visual (top) and tactile (bottom) stimuli during a trial. Each of the squares represents a configuration of the LEDs (small circles) and vibrators (small squares) on the cube. Filled symbols indicate that the LED or vibrator was activated, and empty symbols indicate that the LED or vibrator was turned off.

modality). The second contained 16 single masked target trials $(8$ in each modality). The third consisted of 16 trials that were identical to the experimental trials (two targets), except that the ISI was always $1,500 \mathrm{msec}$.

\section{Results and Discussion}

Accuracy data for T2, which are presented in Figure 2, were submitted to an analysis of variance (ANOVA) including the between-participants variable group (VT vs. TV) and the within-participants variables ISI $(300,450$, 750 or $1,500 \mathrm{msec}$ ) and block type (experimental vs. control). Only the experimental trials in which $\mathrm{T} 1 \mathrm{had}$ been correctly recalled were included. ${ }^{4}$ The analysis re- vealed a significant three-way interaction between group, ISI, and block type $\left[F(3,36)=12.3, M S_{\mathrm{e}}=0.002, p<\right.$ $.001]$, and so we analyzed each group separately.

In the VT group, the main effects of block type $\left[F(1,7)=13.1, M S_{\mathrm{e}}=0.061, p<.01\right]$ and ISI $[F(3,21)=$ $\left.29.9, M S_{\mathrm{e}}=0.003, p<.001\right] \mathrm{reached}$ significance. The interaction between block type and ISI was also significant $\left[F(3,21)=13.7, M S_{\mathrm{e}}=0.003, p<.001\right]$, indicating that accuracy in experimental trials varied in comparison with control trials across ISIs. The effect of ISI was significant for both the experimental block $(p<.001)$ and the control block $(p<.05)$. We analyzed the simple main effects of block type at each ISI, and found differ-
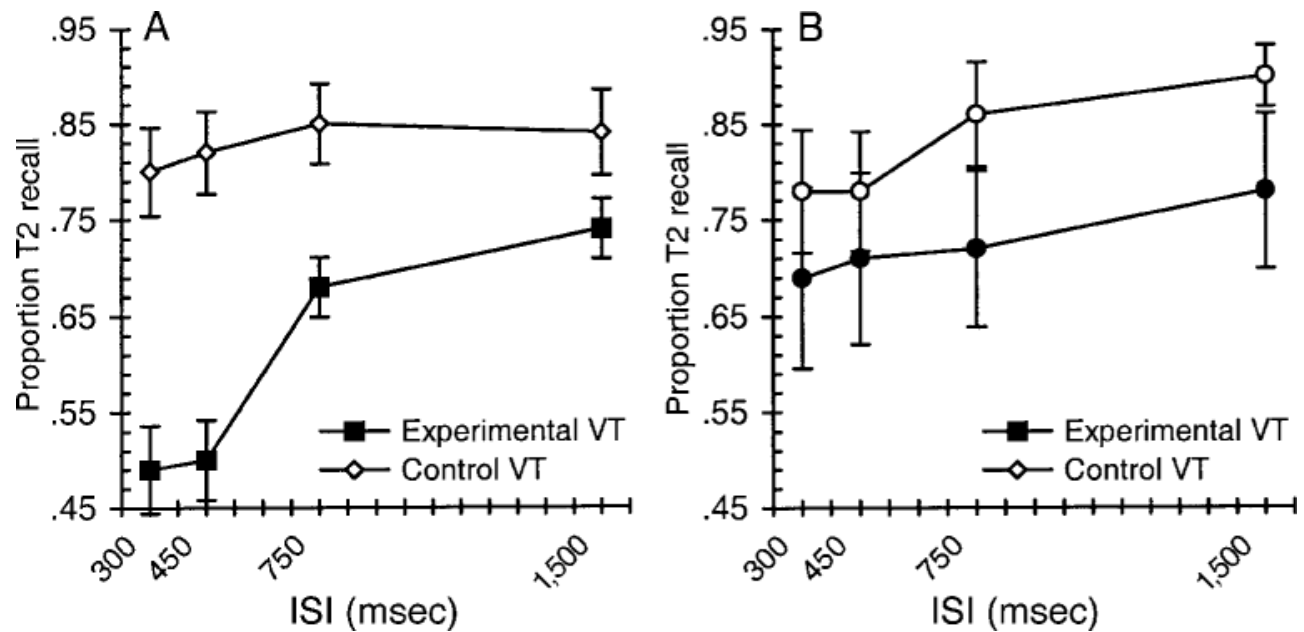

Figure 2. Proportion of correct responses to T2 $( \pm S E)$ as a function of interstimulus interval (ISI) in Experiment 1. Experimental (filled symbols) and control blocks (empty symbols) are presented separately. Only trials on which $\mathrm{T} 1$ was responded to correctly were used to score the trials in the experimental condition. Panel A displays the results for tactile targets preceded by visual T1s. Panel B displays the results for visual targets preceded by tactile $\mathrm{T} 1 \mathrm{~s}$. 
ences at the 300-, 450-, and 750-msec ISIs (all $p$ s $<.05$ ), but not at the 1,500-msec ISI. Thus, there was evidence of an $\mathrm{AB}$ at shorter ISIs.

The ANOVA for the TV group data showed a marginally significant effect of block type $\left[F(1,5)=5.8, M S_{\mathrm{e}}=\right.$ $0.024, p=.06]$, revealing a trend toward more accuracy in control than in experimental trials. The effect of ISI was significant $\left[F(3,15)=6.2, M S_{\mathrm{e}}=0.005, p<.01\right]$ because performance was worse at shorter ISIs. The interaction between block type and ISI was marginally significant $\left[F(3,15)=3.2, M S_{\mathrm{e}}=0.001, p=.052\right]$. As in the VT group, the effect of ISI was reliable for experimental $(p<.05)$ and control $(p<.005)$ blocks. However, in contrast with the results of the VT group, the numerical differences between the control and experimental blocks did not vary significantly as a function of ISI.

The overall advantage (only marginal in the TV group) of control trials over experimental trials (independent of ISI) suggests that it was more difficult to respond to T2 in dual-target than in single-target trials. This dualresponse decrement may be related to a cost associated with participants' switching their attention from the modality of the first target to that of the second (see Quinlan \& Hill, 1999; Spence \& Driver, 1997b; Spence, Nicholls, \& Driver, 2001). Importantly, in the VT group, this decrement was more pronounced at the shortest ISIs, indicating that, on top of any generalized dual-task cost, the processing of $\mathrm{T} 1$ produced a temporary interference in $\mathrm{T} 2$ encoding. This time-locked interference cannot be attributed to task-switching, because the T1 and T2 response sets were identical. Therefore, the results of Experiment 1 provide the first empirical demonstration of a crossmodal $\mathrm{AB}$ between vision and touch. The results of the TV group were different. Other than the overall trend toward a dual-task cost (or crossmodal attention switching cost), the accuracy decrement in the experimental condition was not reduced as ISI increased. Nevertheless, both experimental and control conditions showed a reliable ISI effect. This suggests that the lack of AB (as measured against the "ignore-T1" control condition) in the TV group may be related to potential interference from the irrelevant T1's being just as strong in the control condition as in the experimental condition.

The difference in Experiment 1 between the TV and VT groups is surprising, because it suggests an asymmetry between vision and touch in eliciting a crossmodal AB. Moreover, the fact that the modality of the targets was totally predictable beforehand might have led us to underestimate any possible modality-switching costs in Experiment 1. In the next experiment, we attempted to replicate the finding of Experiment 1 while using a within-participants design and an unpredictable target modality.

\section{EXPERIMENT 2}

\section{Method}

Participants. Twelve volunteers from the pool described in Experiment 1 participated in this experiment. We excluded the data of
1 participant who performed at less than $50 \%$ in the control condition of one modality combination.

Apparatus, Materials, and Procedure. These were the same as in Experiment 1, with the exception that T1 could now be either visual or tactile unpredictably (T2 was always presented in the other modality). Each block contained equal numbers of VT and TV trials. Participants completed six blocks of 128 trials (three experimental and three control, with the sequence counterbalanced across participants). The first two training blocks were as in Experiment 1, and the third was modified to reflect the distribution of materials in Experiment 2.

\section{Results and Discussion}

The average T1 recall accuracy was $.76(S E=3.2)$ in VT trials and $.75(S E=2.9)$ in TV trials. T2 accuracy scores, which are shown in Figure 3, were computed as in Experiment 1 and analyzed in an ANOVA including the within-participants variables target modality (VT vs. TV), block type (control vs. experimental), and ISI (300 vs. 450 vs. 750 vs. $1,500 \mathrm{msec}$ ). Overall, performance was higher in control blocks than in experimental blocks [main effect of block type, $F(1,10)=11.5, M S_{\mathrm{e}}=0.008$, $p<.01]$. Again, performance improved as the ISI increased $\left[F(3,30)=37.5, M S_{\mathrm{e}}=0.009, p<.001\right]$. A significant interaction between block type and ISI was also found $\left[F(3,30)=3.1, M S_{\mathrm{e}}=0.004, p<.05\right]$. The ISI effect was significant for both control and experimental conditions (both $p$ s $<.001$ ). Simple-main-effects analyses of block type at each ISI level showed an AB effect in which performance on experimental trials was worse than that on control trials at the 300-msec ISI $(p<.005)$, but not at the other ISIs (all $p \mathrm{~s}>.05$ ). Although the triple interaction of block type, modality, and ISI was not significant $(F<1)$, we assessed the simple main effects of block type at the 300-msec ISI for each modality combination to parallel the analyses presented in Experiment 1 . Both VT and TV combinations were reliable (both $p \mathrm{~s}<.05$ ). None of the other terms in the ANOVA were significant.

The results from the VT trials replicate the AB effect found in the VT group of Experiment 1. The results from the TV trials demonstrate for the first time that the selection of a tactile target can also lead to a temporary reduction in the ability to process a visual stimulus. However, as in Experiment 1, accuracy was worse at the shortest ISIs even in the control conditions, where the presence of a to-be-ignored $\mathrm{T} 1$ should not affect $\mathrm{T} 2$ performance. There are several possible reasons for this result: First, T1 was the very first event in the trial, and may have reflexively attracted attention; second, T1 could potentially be used by participants as a warning signal to indicate the imminent presentation of $\mathrm{T} 2$, and so could have received some degree of processing; and third, in Experiment 2, knowing that $\mathrm{T} 1$ had been presented was the only way to know which of the two events was T2 (and, moreover, predicted its modality), again inducing participants to pay attention to T1. This may explain why the ISI effect in the control condition was stronger in Experiment 2 than in Experiment 1. For these reasons, the verbal instruction to ignore $\mathrm{T} 1$ may not have 

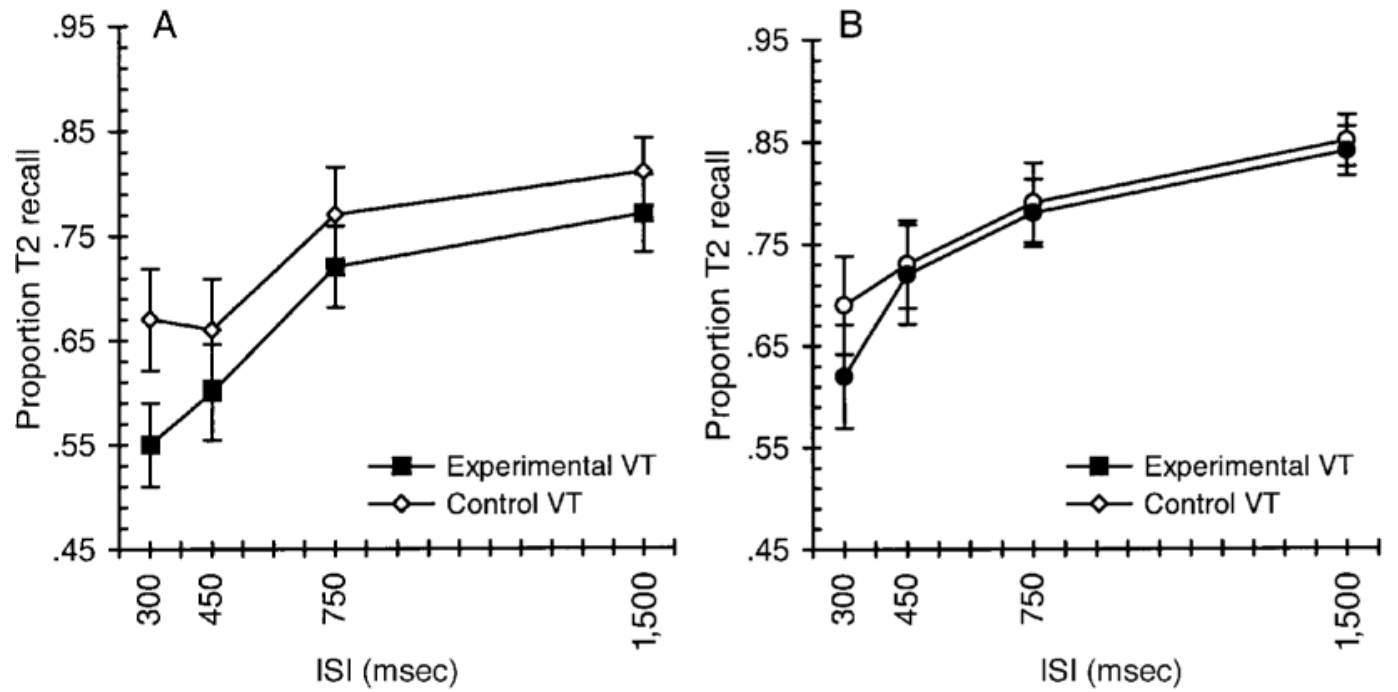

Figure 3. Proportion of correct responses to T2 $( \pm S E)$ as a function of interstimulus interval (ISI) in Experiment 2. Experimental (filled symbols) and control blocks (empty symbols) are presented separately. Only trials in which T1 was responded to correctly were used to score the trials in the experimental condition. Panel A displays the results for tactile targets preceded by visual T1s. Panel B displays the results for visual targets preceded by tactile $\mathbf{T} 1 \mathrm{~s}$.

sufficed to ensure that the first target in the control conditions would be ignored, in turn leading to an underestimation of AB effects (see Chun, 1992, cited in Potter et al., 1998, p. 981, for a similar result).

The primary reason for using the "ignore-T1" task as a control condition in previous $\mathrm{AB}$ studies was to control for sensory forward masking from $\mathrm{T} 1$ as a potential cause of the time-locked interference on T2. However, because we used crossmodal presentation, such concerns about early sensory masking from $\mathrm{T} 1$ on $\mathrm{T} 2$ are less worrisome. To compare $\mathrm{T} 2$ performance in the experimental trials against a baseline that was faithful to its original purpose (i.e., to obtain a measure of performance when attention is fully available), we performed a new analysis using T1 accuracy in experimental trials as the baseline (see Duncan et al., 1994, and R. Ward et al., 1996, for previous studies using this baseline). The data are presented in Figure 4.

We ran an ANOVA including the within-participants variables modality (tactile vs. visual), target (T1 vs. T2), and ISI (300 vs. 450 vs. 750 vs. $1,500 \mathrm{msec}$ ). A significant main effect of target $\left[F(1,10)=15.9, M S_{\mathrm{e}}=0.014\right.$, $p<.005]$ indicated that T2 was recalled less accurately than T1. ISI reached significance $[F(3,30)=17.4$, $\left.M S_{\mathrm{e}}=0.007, p<.001\right]$ because accuracy was lower at shorter ISIs. The interaction between modality and target $\left[F(1,10)=11.8, M S_{\mathrm{e}}=0.004, p<.01\right]$ indicated that the target effect (worse accuracy for T2 than for T1) was stronger for tactile than for visual targets. There was a significant interaction between ISI and target $[F(3,30)=$ $\left.14.5, M S_{\mathrm{e}}=0.005, p<.001\right]$. Simple main effects tests indicated that T1 was not influenced by ISI $(F<1)$, but $\mathrm{T} 2$ performance was worse at shorter ISIs $[F(3,30)=$
23.4, $\left.M S_{\mathrm{e}}=0.008, p<.001\right]$. All of the comparisons between ISIs were significant for T2 (all $p \mathrm{~s}<.05)$. The three-way interaction of modality, target, and ISI was not significant $(F<1)$. This new analysis shows that $\mathrm{T} 2$ performance in experimental trials was strongly dependent on the temporal distance from $\mathrm{T} 1$, whereas $\mathrm{T} 1$ accuracy was unaffected by the temporal separation from $\mathrm{T} 2$.

\section{GENERAL DISCUSSION}

The results of Experiments 1 and 2 demonstrate a crossmodal $\mathrm{AB}$ for tactile stimuli as a function of their temporal distance from a preceding visual target. A crossmodal $\mathrm{AB}$ for visual targets as a function of a preceding tactile stimulus was reported in Experiment 2 (though not in Experiment 1). The present findings contrast with those from previous audiovisual studies, in which no crossmodal audiovisual $\mathrm{AB}$ was observed in the absence of task-switching confounds (e.g., Potter et al., 1998; Soto-Faraco \& Spence, 2002; but note that a clear non-time-locked deficit has been reported; see Spence \& Driver, 1997b; Spence et al., 2001).

\section{Measuring the $\mathrm{AB}$ in the Two-Target Paradigm}

In this study, even the "ignore-T1" conditions produced an ISI-modulated effect on T2 recall accuracy. This dependency suggests that ignoring T1 was simply not possible in the present paradigm (see the discussion of Experiment 2), producing underestimation of $A B$ effects. In support of this view, when we reanalyzed the data using an alternative baseline condition that was stable across ISIs ( $\mathrm{T} 1$ performance), the $\mathrm{AB}$ was more apparent (in both directions, VT and TV). The main reason 


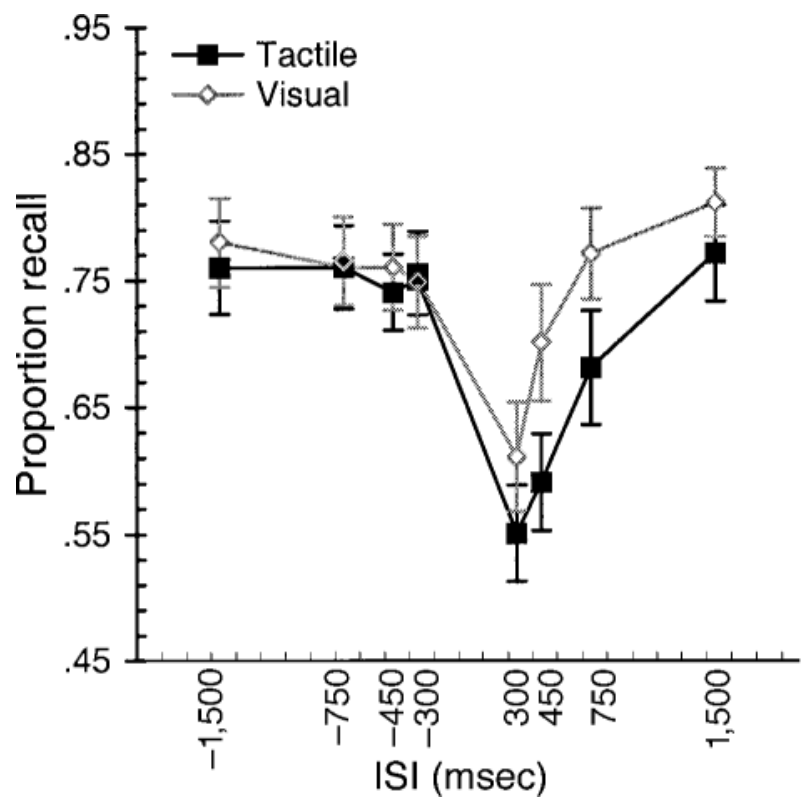

Figure 4. Proportion of correct responses $( \pm S E)$ for both targets of experimental trials in Experiment 2. Accuracy is plotted as a function of the interstimulus interval (ISI) between the targets. Negative ISIs correspond to $T 1$ and positive ISIs to $T 2$. Black squares represent the results for tactile targets (T1 in TV trials and $\mathrm{T} 2$ in VT trials); gray diamonds represent the results for visual targets (T1 in VT trials and T2 in TV trials).

that past studies avoided using T1 report as the baseline was that $\mathrm{T} 1$ was not subject to the same potential sensory forward-masking effects as was $\mathrm{T} 2$. The fact that this study presented targets across different modalities negates this potential problem.

Nevertheless, even if early sensory masking is highly unlikely in visuotactile presentations, one might wonder whether some kind of higher level forward masking from T1 might explain the ISI effects on a different-modality $\mathrm{T} 2$ found in the single-target control group in all conditions. To our knowledge, there is no evidence of forward masking across vision and touch and, even when considered within a single modality (e.g., vision; see Di Lollo, 1980) or across other modalities (e.g., audition and touch; see Gescheider \& Niblette, 1967), forward masking lasts for a period that is far shorter than the ISIs used here. Therefore, we do not believe that forward masking can account for the results, and instead claim that the ISI effect in the control conditions reflects an inability to ignore T1. In any case, independent of unlikely forwardmasking effects, the difference observed between the experimental and control conditions at the shortest ISIs enables us to conclude that a crossmodal visuotactile $A B$ does exist (although it is potentially underestimated in magnitude).

\section{Implications for Theories of the AB}

The prevailing view in the literature is that the $\mathrm{AB}$ reflects a limited-capacity stage of processing (which takes a certain amount of time to complete) following target selection (Chun \& Potter, 1995; Duncan et al., 1997; Potter et al., 1998; Raymond et al., 1992; Shapiro et al., 1994). When $\mathrm{T} 1$ is presented, it undergoes processing in the limited-capacity stage. If $\mathrm{T} 2$ is presented before the completion of $\mathrm{T} 1$ processing, it must wait (for access to the processor), stored in a short-lived representation that is subject to degradation (decay with time, overwriting by trailing items, and competition from distractors have been proposed). The closer together in time two targets occur, the longer the delay in T2 processing and, hence, the greater the chances that $\mathrm{T} 2$ 's stored representation will be degraded and so go unreported. Some researchers (e.g., Potter et al., 1998; Shapiro et al., 1994) have proposed that this limited-capacity processing stage exists only within the visual modality, whereas others (e.g., Duncan et al., 1997) have argued that it can be found between any two same-modality targets but not across modalities. Both views imply that the encoding bottleneck in processing revealed by the $\mathrm{AB}$ does not operate between sensory modalities. However, as noted already, all of the previous crossmodal $\mathrm{AB}$ research has involved only audiovisual presentations. Importantly, the present data indicate that the results from audiovisual studies do not necessarily generalize to other combinations of sensory modalities (specifically, to the visuotactile case studied here).

There are other models that attribute the $\mathrm{AB}$ to interferences occurring at a central stage of processing, during response planning and/or execution (Arnell \& Jolicœur, 1999; Jolicœur, 1999). These can account for, and actually predict, crossmodal $\mathrm{ABs}$ in all possible modality pairings. However, since the crossmodal AB observed here is not enabled by response conflict, the implications of our results for these models are that $\mathrm{AB}$ effects are not necessarily related to interference at the response planning or execution stage. Such a conclusion supports recent claims that what researchers have called an $\mathrm{AB}$ may entail two distinct components (e.g., Arnell \& Duncan, 2002; Potter et al., 1998). For instance, Potter et al. argued that interference effects could originate from responseplanning conflicts and/or from an inability to allocate attention to incoming stimuli. The focus of the present paper has been to isolate attentional interference effects independently of any conflicts at the response stage.

On the basis of the present data, it is clear that current models that explain the $\mathrm{AB}$ interference (in the absence of task-switching) by modality-specific stages in stimulus encoding (e.g., Potter et al., 1998; Raymond et al., 1992) or by modality-specific interference during retrieval (e.g., Shapiro et al., 1994) need to be revised. There are a number of ways in which such a revision could be done. One possibility is to propose that tactile and visual stimuli compete for a common limited-capacity processor, whereas auditory and visual stimuli do not. This proposal makes the prediction that no $\mathrm{AB}$ should be observed between touch and audition ${ }^{5}$ (in the absence of task-switching), because stimuli in these modalities 
would not share a common limited-capacity processor. The implication here is that distinct attentional links may exist between different pairs of modalities, as has been claimed previously on the basis of a variety of empirical crossmodal data (e.g., Spence \& Driver, 1997a; Spence et al., 1998; L.M. Ward, 1994).

However, current theories of the AB can also accommodate the present results if one accepts the idea that the occurrence of a crossmodal $\mathrm{AB}$ is determined by the selection process afforded by the stimuli and/or demanded by the task. For instance, if the stimuli permit and/or the response requires selection on the basis of a shared representation (or attribute), such as position in space, then the $\mathrm{AB}$ would be expected to occur crossmodally (as in the case of the present study). By contrast, if the stimuli permit and/or the response task requires selection on the basis of some modality-specific feature (such as orthographic code in vision and phonological code in audition), then no crossmodal AB would be expected (see Duncan et al., 1997; Potter et al., 1998; Soto-Faraco \& Spence, 2002). The latter account implies that the processing deficit causing the $\mathrm{AB}$ can operate at different levels of representation depending on task demands and/or stimulus characteristics. Note that in this case, no distinctions between attentional links across different pairs of modalities need to be proposed.

\section{REFERENCES}

Arnell, K. M., \& Duncan, J. (2002). Separate and shared sources of dual-task cost in stimulus identification and response selection. Cognitive Psychology, 44, 105-147.

Arnell, K. M., \& Jolicceur, P. (1999). The attentional blink across stimulus modalities: Evidence for central processing limitations. Journal of Experimental Psychology: Human Perception \& Performance, 25, 630-648.

Chun, M. M., \& PotTer, M. C. (1995). A two stage model for multiple target detection in rapid serial visual presentation. Journal of Experimental Psychology: Human Perception \& Performance, 21, 109127.

Dell'Acqua, R., Turatto, M., \& Joliceur, P. (2001). Cross-modal attentional deficits in processing tactile stimulation. Perception \& Psychophysics, 63, 777-789.

Di Lollo, V. (1980). Temporal integration in visual memory. Journal of Experimental Psychology: General, 109, 75-97.

Duncan, J., Martens, S., \& WARD, R. (1997). Restricted attentional capacity within but not between sensory modalities. Nature, $\mathbf{3 8 7}$, 808-810.

Duncan, J., Ward, R., \& Shapiro, K. (1994). Direct measurement of attentional dwell time in human vision. Nature, 369, 313-315.

Gescheider, G. A., \& Niblette, R. K. (1967). Cross-modality masking for touch and hearing. Journal of Experimental Psychology, 74, 313-320.

Hillstrom, A. P. (2000). Repetition effects in visual search. Perception \& Psychophysics, 62, 800-817.

Hillstrom, A. P., Shapiro, K. L., \& Spence, C. (2002). Attentional limitations in processing sequentially presented vibrotactile targets. Perception \& Psychophysics, 64, 1068-1082.

Hughes, H. C. (1999). Sensory exotica: A world beyond human experience. Cambridge, MA: MIT Press.

Joliceur, P. (1999). Restricted attentional capacity between sensory modalities. Psychonomic Bulletin \& Review, 6, 87-92.

Jolicceur, P., \& Dell'Acqua, R. (1999). Attentional and structural constraints on visual encoding. Psychological Research/Psychologische Forschung, 62, 154-164.
Maljkovic, V., \& Nakayama, K. (1994). Priming of pop-out: I. Role of features. Memory \& Cognition, 22, 657-672.

McDonALD, J. J., \& WARD, L. M. (1999). Spatial relevance determines facilitatory and inhibitory effects of auditory covert spatial orienting. Journal of Experimental Psychology: Human Perception \& Performance, 25, 1234-1252.

Pallier, C., Dupoux, E., \& Jeannin, X. (1997). EXPE: An expandable programming language for on-line psychological experiments. $B e$ havior Research Methods, Instruments, \& Computers, 29, 322-327.

Potter, M. C., Chun, M. M., Banks, B. S., \& Muckenhoupt, M. (1998). Two attentional deficits in serial target search: The visual attentional blink and an amodal task-switch deficit. Journal of Experimental Psychology: Learning, Memory, \& Cognition, 24, 979-992.

Quinlan, P. T., \& Hill, N. I. (1999). Sequential effects in rudimentary auditory and visual tasks. Perception \& Psychophysics, 61, 375-384.

RaYmond, J. E., Shapiro, K. L., \& Arnell, K. M. (1992). Temporary suppression of visual processing in an RSVP task: An attentional blink? Journal of Experimental Psychology: Human Perception \& Performance, 18, 849-860.

Shapiro, K. L., Raymond, J. E., \& Arnell, K. M. (1994). Attention to visual pattern information produces the attentional blink in rapid serial visual presentation. Journal of Experimental Psychology: Human Perception \& Performance, 20, 357-371.

Soto-Faraco,S., \& Spence, C. [J.] (2002). Modality-specific auditory and visual temporal processing deficits. Quarterly Journal of Experimental Psychology, 55A, 23-40.

SPEnCE, C. [J.] (2001). Crossmodal attentional capture: A controversy resolved. In C. Folk \& B. Gibson (Eds.), Attraction, distraction, and action: Multiple perspectives on attentional capture (pp. 231-262). Amsterdam: Elsevier.

SPENCE, C. J., \& DRIVER, J. (1994). Covert spatial orienting in audition: Exogenous and endogenous mechanisms facilitate sound localization. Journal of Experimental Psychology: Human Perception \& Performance, 20, 555-574.

SPEnce, C. [J.], \& Driver, J. (1997a). Audiovisual links in exogenous covert spatial orienting. Perception \& Psychophysics, 59, 1-22.

Spence, C. [J.], \& Driver, J. (1997b). On measuring selective attention to a specific sensory modality. Perception \& Psychophysics, 59, 389403.

Spence, C. [J.], Nicholls, M. E. R., \& Driver, J. (2001). The cost of expecting events in the wrong sensory modality. Perception \& Psychophysics, 63, 330-336.

Spence, C. [J.], Nicholls, M. E. R., Gillespie, N., \& Driver, J. (1998). Cross-modal links in exogenous covert spatial orienting between touch, audition, and vision. Perception \& Psychophysics, 60, 544-557.

Spence, C. [J.], Pavani, F., \& Driver, J. (2000). Crossmodal links between vision and touch in covert endogenous spatial attention. Journal of Experimental Psychology: Human Perception \& Performance, 26, 1298-1319.

Stein, B. E., \& Meredith, M. A. (1993). The merging of the senses. Cambridge, MA: MIT Press.

WARD, L. M. (1994). Supramodal and modality-specific mechanisms for stimulus-driven shifts of auditory and visual attention. Canadian Journal of Experimental Psychology, 48, 242-259.

Ward, R., Duncan, J., \& Shapiro, K. (1996). The slow time course of visual attention. Cognitive Psychology, 30, 79-109.

Ward, R., Duncan, J., \& Shapiro, K. (1997). Effects of similarity, difficulty, and nontarget presentation on the time course of visual attention. Perception \& Psychophysics, 59, 593-600.

\section{NOTES}

1. The term task-switching is used to denote those dual-target procedures in which the response set of the first target is different from that of the second target. This definition is widely used in the literature (e.g., Potter et al., 1998; Quinlan \& Hill, 1999). Alternative definitions of task-switching might include situations in which a switch in the defining feature of the targets occurs even in the absence of a change in the response feature (e.g., Hillstrom, 2000; Maljkovic \& Nakayama, 1994). However, we believe that the former definition is more appropriate in 
the present context, given that the latter would not allow one to categorize previous crossmodal $\mathrm{AB}$ research in an informative way (by definition, all crossmodal trials would include a task switch).

2. The complex pattern mask for visual targets, as well as the fact that visual stimuli consisted of offsets instead of onsets, was designed to avoid the sensation of flow from the stimulated corner that these kinds of stimuli can sometimes create.

3. Given that the number of SOA levels that we could test was limited by the length of the experiment, we decided not to include very short SOAs in order to bypass any potential lag-1-sparing phase of the AB (see Raymond et al., 1992; Shapiro et al., 1994). On the basis of previous pilot work, we included SOAs at which a maximum $A B$ was expected (lags 2 and 3 ) and SOAs at which performance should have recovered from the $\mathrm{AB}$ (lags 5 and 9). Lag was defined as the length of one target-mask combination $(150 \mathrm{msec})$.
4. Mean T1 recall accuracy in the experimental condition was $80 \%$ $(S E=2.8)$ in the TV group. Unfortunately, the corresponding data for the VT group were lost. However, the training session ensured that the participants were able to perform at above $70 \%$ correct for $\mathrm{T} 1$ report.

5. During the publication process, we became aware of a recent study by Dell'Acqua, Turatto, and Jolicœur (2001), in which a temporary interference between tactile and auditory tasks was reported. It is important to note that, because the paradigm used by Dell'Acqua et al. contained a switch between the tactile and the auditory tasks, the implications for present discussion are only marginal.

(Manuscript received June 6, 2000; revision accepted for publication January 3, 2002.) 